\title{
Research into Quantum Measurement and De-coherence
}

\author{
Zhiguo Wang \\ School of Physics and Electronic and Electrical Engineering of Huaiyin Normal University, Huai 'an, \\ Jiangsu Province, 223300
}

KEYWORD: quantum measurement; quantum information; entangled state; quantum de-coherence ABSTRACT: Measurement is a very important object of quantum information and quantum computation theory, and an important tool for obtaining quantum information. The development of quantum measurement theory not only plays a very important role in the field of quantum theory, but also gradually penetrates into other inter-disciplines. This thesis introduces the theory of quantum measurement and communication, explores the features of quantum teleportation and privacy communication, and addresses the issue of quantum de-coherence in the process of measurement, which can offer some beneficial enlightenment to us in acquiring a further understanding of the theory of quantum communication.

\section{INTRODUCTION}

Measurement is a very important object of quantum information and quantum computation theory, and an important tool for obtaining quantum information ${ }^{[1]}$. Before and after the measurement of quantum system, quantum state changes, hence triggering off a series of major problems, e.g. EPR paradox, Bell inequation, etc. The development of quantum measurement theory not only plays a very important role in the field of quantum theory, but also gradually penetrates into other inter-disciplines $^{[2]}$. In 1997, Zeilinger group of Austria completed the theoretical experimental verification of teleportation of quantum state indoors for the first time ${ }^{[3]}$. In 2004, this group successfully increased the distance of teleportation of quantum state to 600 meters by using the optical fiber channel in the bottom of the Danube. In 2004, Pan Jianwei, Pen Chengzhi and other research fellows of University of Science and Technology of China began to explore how to achieve long-distance quantum communication in a free space ${ }^{[4]}$. In 2005, this group created a record of the double quantum entangled distribution in a free space for a distance of $13 \mathrm{~km}$ in Hefei, Anhui Province, China, and verified the feasibility of distributing entangled photons between the outer space and the earth at the same time. Since 2007, the United Research Group of Tsinghua University and University of Science and Technology of China has set up a 16-km free space quantum communication channel between Badaling, Beijing and Huailai, Hebei Province, made a series of key technological breakthroughs, and eventually successfully achieved long-distance teleportation of quantum state for the first time in the world in 2009. This has not only verified the feasibility for the teleportation of quantum state to pass through the atmosphere, but also laid a solid foundation for the globalized quantum communication network among future satellites. As anticipated, globalized quantum communication can be put into effect within 10 years, which is of great revolutionary significance for the future network communication development and information processing.

\section{THEORY OF QUANTUM COMMUNICATION}

Quantum communication mainly makes use of the quantum characteristics of light in the micro-world, and achieves the high-speed transmission of information with single photons or entangled photons as information carriers. Theoretically, quantum communication enables the transmission of information of ultra-large capacity, the generation of a secret key which cannot be deciphered, and the enciphering of data and information with this secret key for communication. 


\section{Quantum teleportation}

In 1993, Bennet et al published a ground-breaking article on PRL, in which they put forward the scheme of teleportation of quantum state: the unknown quantum bit of a particle is transmitted to another place, and another particle is prepared on this quantum state, while the original particle is still kept in its original place. His basic idea is: the information of the original substance is classified into two types: quantum information and classic information, and they are transmitted to receivers via the quantum channel and classic channel. Quantum information is the remaining information which the transmitters fail to extract during measurement, while classic information is acquired by transmitters by carrying out a certain kind of measurement to original substances. After acquiring these two types of information, receivers can produce the replicas of quantum states of original things. What are transmitted in this process are not original substances themselves, but the quantum states of original substances. Transmitters can be even ill-informed of the quantum states, while receivers transform other material units into the same quantum states as those of original substances, and the quantum states of original substances have already been destroyed in this process. Quantum teleportation is of great significance for people to understand and reveal the principles of the natural world in the field of physics. Moreover, by using quantum states as information carriers, we can complete information transmission of ultra-large capacity through quantum state teleportation, and can achieve quantum privacy communication which cannot be deciphered in principle.

\section{Quantum privacy communication}

Privacy is always a problem in the telecommunication field. Secret keys are universally used by the privacy techniques which are applied at present, requiring both parties to have the same code book. However, the code book is unsafe in the process of transmission, because it may be eavesdropped or duplicated by others. Therefore, the present privacy communication is unsafe in essence. The theory of classic privacy communication is shown in fig 1 . Before secret keys are established for quantum communication, there is no code book, but an agreement has been reached with the other party. The properties of quantum are used through this agreement. These properties are known ones and are open to the public. The light quantum mentioned in quantum communication is the basic composition unit of rays: photon. Photons are transmitted to the other party via optical fibers or rays. Secret keys of quantum are random series which express "0" or " 1 " via different quantum states attributed to polarization modulation or phase modulation of single photons. The random series are also called quantum bits. After the random number is transmitted to the other party, a random secret key would come into being as soon as the other party receives the random number. The secret key is established only when needed, and it needn't be kept, thus avoiding being revealed. The process of eavesdropping is equivalent to a process of measurement. Once eavesdroppers carry out an eavesdropping operation, an error code would be brought into the process of quantum communication, thus increasing the rate of error codes, and leading to discovery.

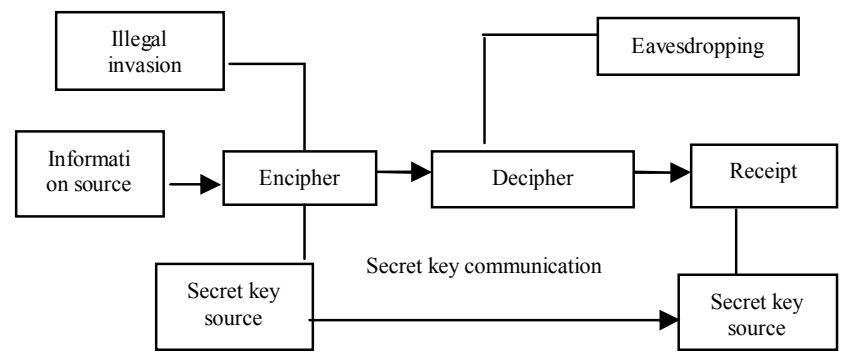

Fig 1: The schematic diagram of classic privacy communication.

\section{QUANTUM MEASUREMENTS AND QUANTUM DE-COHERENCE}

\section{Quantum measurement hypothesis}

In order to acquire all types of the information about the law of motion of micro-system, we must carry out measurement according to wave function and with the help of classic instruments. In order to explain the measurement issue more explicitly, we take the double-slit experiment in quantum 
measurement for an example. When particles are measured, the interference fringes would come into being on the screen when particles pass through double slits. Before measurement, particles are coherent, and this quantum coherence is a fundamental attribute of the micro-world. When it is detected that a particle arrives at the screen through a slit, the interference fringe on the screen would disappear. Such a phenomenon as deprives particles of coherence is called quantum de-coherence, and the quantum de-coherence attributed to measurement is called the collapse of the wave packet. Therefore, quantum measurement is closely related to quantum de-coherence. Therefore, it is necessary for us to make a precise definition to and work out a series of relevant regulations for this kind of measurement. The so-called quantum measurement means producing a kind of interaction between the measured system and the measuring instruments, thus enabling us to get to know the state of the measured system from the state of measuring instrument.

\section{The issue of quantum de-coherence in the process of measurement}

Quantum coherence is a universal attribute of the motion in the micro material world. However, the effective measurement of micro particle motion state will certainly produce an irreversible change over the original motion of particle, resulting in the destruction of quantum coherence. Take the double-slit experiment of material wave interference for an example. If the measured particle passes through a slit, the particle feature of wave-particle dualism is emphasized. Wave motion property is rejected as the complementation of particle property, and interference fringe no longer exists. Because wave is an entity which permeates the entire space, point particle can nevertheless be localized to one of the two slits. This discussion about the disappearance of interference fringe is based on the complementary theory of quantum mechanics or the principle of complementarities under the standard "Copenhagen interpretation": material motion has a double attribute of particle and wave, but the two repel each other in the same experiment.

This phenomenon of disappearance of coherence resulting from measurement or any other influence is called "quantum de-coherence". If only quantum measurement is concerned, it is called the collapse of the wave packet. The further interpretation to this phenomenon of de-coherence is the application of the uncertainty relation of Heisenberg: Accurately knowing that a particle passes through Path A means fully defining the location of the particle which is accurate to $\Delta \mathrm{x}$ in the direction perpendicular to $\mathrm{A}$; we can also learn from the uncertainty relation principle $\Delta x \cdot \Delta p \sim$

$\hbar$ that this measurement will produce a disturbance of $\Delta p \sim \hbar / \Delta x$ to the momentum in the direction perpendicular to Path A. When its interference arrives at the location of the particle on Screen $\mathrm{S}$, it will make the interference fringe vague. In this sense, the uncertainty relation indicates that describing the material motion of wave-particle duality with the measurement about particle characteristics will cause the uncertainty of measurement.

\section{The significance of quantum de-coherence}

The above-mentioned discussion about de-coherence is based on the hypothesis of conducting a single measurement and the requirement that results must be read out. At this time, wave function is reduced to one of its branches. This result is called "Type 1 collapse of the wave packet". However, wave function is related to specific experiment through statistic interpretation, namely: the result of prediction which can be distinguished macroscopically can be obtained through several rounds of single measurement.

In this sense, we need to bring in the concept of Type 2 collapse of the wave packet. We can assume that we measure the ensemble of the system, instead of carrying out a round of measurement against a single quantum system and reading out an exact result. For this purpose, we should apply the concept of density matrix to describe the post-measurement system state. When we measure the system located in $|\Psi\rangle=\Sigma \infty \mathrm{n}=0 \mathrm{cn}|\mathrm{n}\rangle$, quantum mechanics fails to tell us the exact result of 
measurement. It only predicts that the probability of obtaining "an" by measuring on $|\Psi\rangle$ is $|\mathrm{cn}| 2$. Therefore, positive definite operator-density matrix operator $\rho \mathrm{f}=\Sigma \mathrm{n}|\mathrm{cn}| 2|\mathrm{n}\rangle\langle\mathrm{n}|$ describes the classic probability state of post-measurement system. The quantum de-coherence process represented by collapse of the wave packet can be denoted as the disappearance process of non-opposite-angle item of transformation from initial state density matrix $\rho=|\Psi\rangle\langle\Psi|$ to $\rho f$. This is the so-called Type 2 collapse of the wave packet. Usually, it indeed represents the disappearance of coherence and even coherent fringe.

\section{CONCLUSIONS}

As can be inferred from the above-mentioned discussion, the research into quantum measurement and its related de-coherence issue are not only of great theoretical significance, but also boast a huge potential of application in quantum information. In terms of the basic concept, the research into quantum measurement and its related quantum de-coherence involves a more universal essential issue of modern physics. The Schrodinger equation is a basic one which describes the motion of micro-particles. Under the transformation of time reversal, it remains unchanged, thus being symmetrical in terms of time reversal. Therefore, the single particle process described by the Schrodinger equation is micro-reversible. However, in the macro and classic world, macro-objects composed of these particles demonstrate an irreversible classic behavior. The transformation from micro-reversibility to macro- irreversibility is quite complicated. How to coordinate macro-irreversible phenomena and basic quantum mechanics is concerned with everyone and remains an unsolved problem.

\section{ACKNOWLEDGEMENTS}

This work was financially supported by the project under the National Spark Program (2011GA690403)and the project of research on the higher educational reform of Huaiyin Normal University(11GJA0020):The Practice and Innovation Projects for university students of Jiangsu (201310323058X).

\section{BIBLIOGRAPHIES}

[1] Zhou Zhengwei, Tu Tao et al; The Progress and Prospect of Quantum Computation[J].The Progress of Physics, Vol.29(Jun.2009) No.3,p.127.

[2] Zhang Yongde. Principles of Quantum Information Physics [M].Beijing: Higher Education Press, 2006.1:258-276.

[3]D. Bouwmeester, J. W. Pan, K. Mattle et al. ,Nature,390(1997),575.

[4]Pan J W, Gasparoni S,Aspelmeyer M,et al. Experimental realization of freely propagating teleported qubits[J]. Nature, 2003, 421(6924): 721-725.

[5] Bennett C H,Brassard G,Crepeau C,et al. Phys Rev Lett,1993,70 (13 ):1895-1899.

[6] Einstein A, Podolsky B , Rosen N. Phys Rev , 1935 , 47 :777.

[7]Bouwmesster D,Pan J W,Mattle K.Experimental quantum teleportation[J].nature, 1997,390:575

[8]Vaidman L. Teleportation of quantum states[J]. Phys Rev A,1994,49:1473-1476.

[9]Furusawa A, Sorensen J L, Braunstein S L. Unconditional quantum teleportation[J].Science, 1998,282:706.

[10]D. Boschi et al., Phys. Rev. Lett. , 80 (1998),1121.

[11] de Riedmatten H, Marcikic I, Tittel W, et al.Long distance quantum teleportation in a quantum relay configuration[J ].Phys Rev Lett , 2004,92(4):047904.

[12] Zhang Q, Goebel A, Wagenknecht C et al.Nature Physics, 2006, 2: 678

[13] Chen Y A, Chen S, Yuan Z S et al. Nature Physics, 2008,4:103

[14] Wang Zhiguo. Characteristics of Phase Transition of Bose-Einstein Condensation in Momentum Space. Recent Advance in Statistics Application and Related Areas.Vo.1(2009)p.361-341.

[15] Yu Hua-Ling, Wang Zhi-Guo,et al.Phase Coherence of the Electron and Hole in a Ferromagnetic Film in Proximity with a Superconductor[J]. Chin. Phys.B. 
2008.17(12)4627-4634. 